\title{
Are Selective Serotonin Reuptake Inhibitors a Secondary Cause of Low Bone Density?
}

\author{
Kim Chau, ${ }^{1}$ Stephanie A. Atkinson, ${ }^{1}$ and Valerie H. Taylor ${ }^{2,3}$ \\ ${ }^{1}$ Department of Paediatrics, McMaster University, Hamilton, ON, Canada L8S 4 L8 \\ ${ }^{2}$ Department of Psychiatry, University of Toronto, Toronto, ON, Canada L8S $4 \mathrm{~L} 8$ \\ ${ }^{3}$ Women's College Hospital, 76 Grenville Street, Toronto, ON, Canada M5S 1 B2 \\ Correspondence should be addressed to Valerie H. Taylor, valerie.taylor@wchospital.ca \\ Received 19 October 2010; Accepted 18 December 2011 \\ Academic Editor: D. Felsenberg
}

Copyright (c) 2012 Kim Chau et al. This is an open access article distributed under the Creative Commons Attribution License, which permits unrestricted use, distribution, and reproduction in any medium, provided the original work is properly cited.

\begin{abstract}
Background. Osteoporosis is a chronic disease that can significantly impact numerous aspects of health and wellness. The individual consequences of osteoporosis can be devastating, often resulting in substantial loss of independence and sometimes death. One of the few illnesses with greater disease burden than low bone mineral density (BMD) is major depressive disorder (MDD). Both depression and antidepressant use have been identified as secondary causes of osteoporosis. The objective of this paper is to review and summarize the current findings on the relationship between antidepressant use and BMD. Methods. Relevant sources were identified from the Pubmed and MEDLINE databases, citing articles from the first relevant publication to September 1st, 2010. Results. 2001 articles initially met the search criteria, and 35 studies were thoroughly reviewed for evidence of an association between SSRI use and BMD, and 8 clinical studies were detailed and summarized in this paper. Conclusions. Current findings suggest a link between mental illness and osteoporosis that is of clinical relevance. Additional longitudinal studies and further research on possible mechanisms surrounding the association between SSRI use on bone metabolism need to be conducted. Treatment algorithms need to recognize this association to ensure that vulnerable populations are screened.
\end{abstract}

\section{Introduction}

Osteoporosis is a chronic disease that affects approximately $26 \%$ of women aged 65 years or older $[1,2]$. A 50 -yearold woman has approximately a $40 \%$ chance of sustaining an osteoporotic fracture $[3,4]$, and a 14 -year-old girl has a $17 \%$ chance of sustaining a hip fracture at some point in her lifetime [5]. The individual consequences of osteoporosis can be devastating, often resulting in substantial loss of independence and sometimes death [6]. The burden on the health care system is also substantial, and it is estimated that the annual cost of hip fractures could exceed $\$ 2.4$ billion by 2041 [7]. It is also an illness that is preventable if identified early and managed appropriately.

One of the few illnesses with greater disease burden than low bone mineral density (BMD) is major depressive disorder (MDD); it has been projected that MDD will be the biggest cause of disability world wide by 2020 [8]. Importantly, this is not simply attributed to psychiatric morbidity, and, in fact, MDD has been linked to a host of physical illnesses, mitigated in large extent by side effects of pharmacotherapy [9]. Recent evidence highlights the fact that impaired bone health may soon be joining this growing list.

1.1. Evidence of an Association between Antidepressant Use and Bone Health. Serotonin (5-hydroxytryptamine; 5-HT) is a neurotransmitter produced primarily in serotonergic neurons in the central nervous system (CNS). Its primarily role is to influence psychological and behavioural functions such as mood, anxiety, and sleep, and, as a consequence, it is a key player in the pathophysiology of MDD and other psychiatric illnesses [10-14]. Therefore, not surprisingly, a wide range of psychiatric disorders are treated with drugs that target this system [14-17].

Selective serotonin reuptake inhibitors (SSRIs) represent a class of medications that selectively and potently block the serotonin transporter (5-HTT) in the CNS to 
effectively increase the extracellular levels of serotonin and relieve symptoms of depression [12]. The primary target of SSRIs are serotonin transporters (SERT), and the general principle of the SSRIs' mechanism of action is to boost the synaptic activity of serotonin by acute pharmacological inhibition of presynaptic SERTs, thereby increasing the synaptic concentration and activity of serotonin $[12,18-$ 22]. As a result of elevated central serotonin, depressive symptoms are alleviated. The impact of serotonin 5-HT is not confined to the CNS, however, and a functional 5-HT signaling system in bone was identified in 2001 [23, 24]. Further investigation of this peripheral 5-HT system has also shown that SSRIs appear to affect both CNS and bone 5-HTT with similar potency [23]. While the specific biochemical nature of serotonergic pathways and their direct and/or indirect effects on bone metabolism are still unclear, existing data suggest an association between depression and increased risk of fracture and bone loss that may be mediated in part by antidepressants [16].

\subsection{The Role of Serotonin in Bone Health. A functional} role for 5HT in bone was first documented in 2001 when Bliziotes and colleagues demonstrated the presence of neurotransmitters, receptors, and transporters in osteoblasts and osteoclasts $[23,25,26]$. This group documented that the serotonergic system in bone plays a critical role in bone metabolism, a fact that was later confirmed by Westbroek and colleagues $[23,24]$. Their findings also revealed that knockout mice without the serotonin transporter demonstrated significant decreases in bone density, impaired bone architecture, and bone mechanical properties [23]. Bliziotes' group also suggested that one possible mechanism to explain 5HT's negative effect on bone is the reduction in osteoblast activity, as a result of serotonin transporter inhibition, leading to lower BMD. This work provided evidence of the role serotonin in bone metabolism and a mechanism through which SSRIs may influence bone health [23]. Work on the link between depression, SSRI use, and BMD is equivocal; however, no association was noted between antidepressant use and BMD among men and women 17 years of age and older using data from the Third National Health and Nutrition Examination Survey (NHANES III) [27] or among postmenopausal women participating in the Women's Health Initiative (WHI) Observational Study [28]. In contrast, a recent meta-analysis identified depression, and especially depression in premenopausal women, as a significant risk factor for low BMD [29], while new work on novel mechanisms of serotonergic modulation of bone mass [30] highlights the biologic plausibility of an antidepressant mediated mechanism of decreased BMD.

The risks of low BMD associated with serotinergic antidepressant use and/or depression needs to be clarified. The scope of this problem cannot be denied; the use of antidepressants is ubiquitous, with combined American sales that exceeded $\$ 10$ billion in 2004 [37]; a report published in 2000 ranked antidepressant drugs third among all drugs in the US prescription drug sales [38] and listed SSRIs as the most commonly prescribed class of antidepressants, accounting for approximately $62 \%$ of all antidepressants prescribed in the United States in 2002 [39]. Therefore, the goal of this paper is to summarize the literature on the association between SSRI use and bone health.

\section{Materials and Methods}

2.1. Study Selection. A comprehensive literature search using the computerized databases Pubmed and MEDLINE to identify relevant studies, covering the period ending September 2010, was performed using the medical subject headings "selective serotonin reuptake inhibitors," "depression," "major depressive disorder," "antidepressants," "bone mineral density," "osteoporosis," and "hypothalamic-pituary adrenal axis.” A manual search of relevant reports was conducted by examining reference lists from original research papers and review articles. An initial screening was made of titles and abstracts of the articles, and simple relevant criteria for human participants, antidepressants, SSRIs, MDD, and BMD were used to exclude obviously irrelevant references. Inclusion criteria were (1) English-language journals, (2) full published studies with original data in peer-reviewed journals, (3) confirmation of depression with a standardized diagnostic tool, and (4) treatment with SSRIs. In total, eight relevant studies were identified that complied with these search criteria $[16,17,31-36]$.

2.2. Data Extraction. All data were extracted independently by two investigators (MKC and VHT) using a standard protocol and data-collection form. Disagreements were resolved by discussion and, when necessary, by additional input from a third investigator. The extracted information included name of first author, year of publication, study design, country where the study was conducted, characteristics of study subjects (sample size, sampling methods, and distribution according to gender, mean age, race, weight, body mass index (BMI), menopausal status (women only), and antidepressant medication history), measures of BMD and of depression, confounding factors that were controlled by matching or multivariate adjustment, and mean BMD for depressed SSRI-treated, nontreated and nondespressed persons (if possible).

\section{Results}

Our initial search on PubMed identified 2001 potentially relevant and eligible studies; 35 full text articles were retrieved and screened for more detailed evaluation. Redundant references were eliminated, and studies that did not meet the eligibility criteria were excluded; therefore, a total of eight articles remained [16, 17, 31-36].

The eight articles reviewed in detail were human clinical studies [16, 17, 31-36]. Six studies examined BMD in SSRI users compared to nonusers and suggested an association between SSRI use and lower age- and gender- related BMD in humans $[16,17,31,32,34,35]$. Richards et al. along with Ziere et al. also examined the fracture risk in SSRI users compared to nonusers. These studies suggested an 
association between SSRI use and risk of fractions; however, only one study investigated BMD concomitantly with the risk of falls [17]. Of the eight studies identified, seven studies used fan-beam dual energy X-ray absorptiometry (DXA; QDR $4500 \mathrm{~W}$, Hologic Inc.) to measure BMD (g/cm2) [16, 17, 3135 ] and one used the dual energy X-ray absorptiometry pencil beam (DPX-L, Lunar Corp., Madison, WI) [36].

There were various methods applied for assessing depressive symptoms. Five studies used the Diagnostic and Statistical Manual of Mental Disorder (DSM) depression assessment criteria [31-35], one study [36] used criteria for depression according to the Center for Epidemiologic Studies Depression Scale (CES-D), and others used the Mental Component Score (MCS), the Mental Health Inventory 5 (MHI-5) scales of the Medical Outcomes Study 36-Item Short-Form Health Survey questionnaire [17], or the Geriatric Depression Scale (GDS) [16].

Of the eight human clinical studies reviewed, five studies clearly provide support for an association between treatment with SSRI and lower BMD [16, 17, 32, 34, 36]. In contrast, three small studies demonstrated no connection between SSRI treatment and lower BMD [31, 33, 35]. Sample sizes in the studies selected ranged from 42 to 7983 and, and six studies were case-control studies and two contained data from prospective cohorts (Table 1).

\section{Discussion}

4.1. Clinical Studies Investigating an Association between SSRI Use and Bone Health. The possibility of an association between SSRI use and low BMD has sparked a recent rise in studies investigating the clinical implications of antidepressant treatment on bone health. In 2005, Cauley and colleagues conducted a population-based cross-sectional study of participants enrolled in The Osteoporotic Fractures Study in Men (aged 65 years) to determine the factors associated with BMD of the lumbar spine and proximal femur. The authors concluded that SSRI use was independently associated with a lower spine and hip BMD [32] but it was noted that the weight loss and poor diet in persons with depression could have confounded the results observed. A separate study was conducted by Diem et al. to determine whether SSRI use in a cohort of 2744 women (65 years) enrolled in the Study of Osteoporotic Fractures was associated with increased rates of bone loss, specifically in the hip. Patients in the study were divided into either "partial users" where SSRI use was recorded at one of the two visits only or "recurrent users" where SSRI use was recorded at both visits [16]. The study covered a period of 4.9 years, and BMD of the total hip and 2 subregions (femoral neck and trochanter) was assessed with serial measurements over 2 separate visits. The end result was that SSRI use in women was independently associated with increased rates of hip bone loss compared to nonusers [16]. An analysis of the Canadian Multicentre Osteoporosis Study (CaMos) cohort revealed an association between SSRI use and lower BMD that was related to increased clinical fragility fracture risk [17]. Consistent with the findings revealed by Richards et al. [17] and Diem et al. [16], the results from a large cohort study conducted in Rotterdam demonstrated that the use of SSRIs was associated with a 2.25-fold increase in fracture risk [36]. Of note, the authors in this study were able to distinctly define a direct correlation between treatment duration and greater fracture risk, which was detectable as early as 6 weeks following treatment. Furthermore, a similar trend was observed in an observational study conducted by Williams et al. to investigate the effect of SSRIs on BMD in women with a lifetime history of depressive disorder (SSRItreated group and untreated) [34]. The results indicated that BMD among SSRI users was 5.6\% ((0.977 (0.116) versus $\left.\left.0.922(0.117) \mathrm{g} / \mathrm{cm}^{2}, P=0.03\right)\right)$ lower at femoral neck, $6.2 \%$ $\left(0.813(0.105)\right.$ versus $\left.\left.0.763(0.107) \mathrm{g} / \mathrm{cm}^{2}, P=0.04\right)\right)$ lower at the trochanter, and $4.4 \%((0.745(0.007)$ versus 0.712 $\left.(0.068) \mathrm{g} / \mathrm{cm}^{2}, P=0.03\right)$ ) lower at mid-forearm compared to SSRI nonusers [34]. Based on these findings, Williams and colleagues concluded that SSRIs negatively impacts BMD independent of the effect of depression on bone health. Of the above-mentioned studies, Williams et al. is the only study in which depressed patients were diagnosed according to the DSM-IV [34]. Interestingly, according to Bab and Yirmiya, the strength of an association is stronger, displaying significantly lower BMD, when patients were diagnosed with MDD by clinical assessment as opposed to being diagnosed by self-rated questionnaires [40].

Inconsistency exists in observations of an association between SSRI use and reduced BMD, with three small studies demonstrating a relationship $[31,33,35]$. In a study of the association between depression and BMD, 24 women with past or current MDD were matched with 24 healthy controls, with 15 of the depressed women reporting SSRI use [31]. Those women with current or past depression had lower trabecular bone density as compared to healthy controls, but, after controlling for age and BMI, BMD did not correlate with the duration of antidepressant drug therapy. The authors ultimately reported no association between a lifetime use of antidepressant drug treatment and bone density [31]. Similarly, Eskandari and colleagues conducted a prospective study in premenopasal women in which they examined the association between MDD and BMD using immune, pituitary-adrenal, and sympatheticbiomarkers to determine whether this population had a higher prevalence of osteopenia and osteoporosis and lower BMD than healthy controls [33]. While an association between premenopausal women with MDD and lower bone mass was confirmed, like the Michelson study, no association was reported between SSRIs use and BMD [33]. A limitation of this study as indicated by the authors is that women with MDD in their cohort had approximately $5 \mathrm{~kg}$ higher body mass than in other studies cohorts. This may have resulted in the lack of an association between SSRI use and BMD, given that higher body mass positively affects BMD [33]. Further support for these observations is found in the crosssectional study of premenopausal women with unipolar depression matched with healthy controls who demonstrated significantly lower BMD. After adjusting for duration of drug exposure, however, it appeared that antidepressants had no impact on the osteodensitometric results [35]. In contrast to 


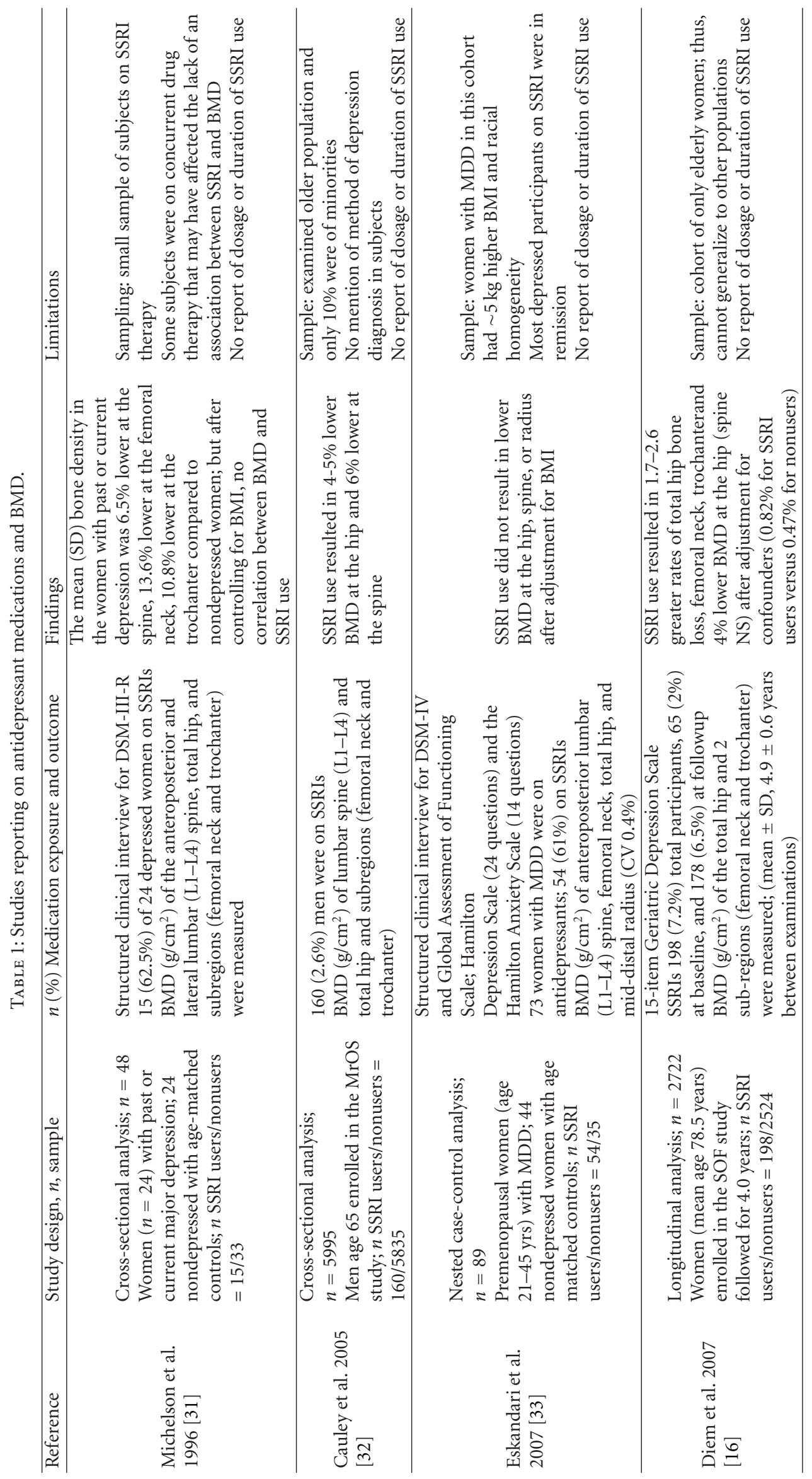




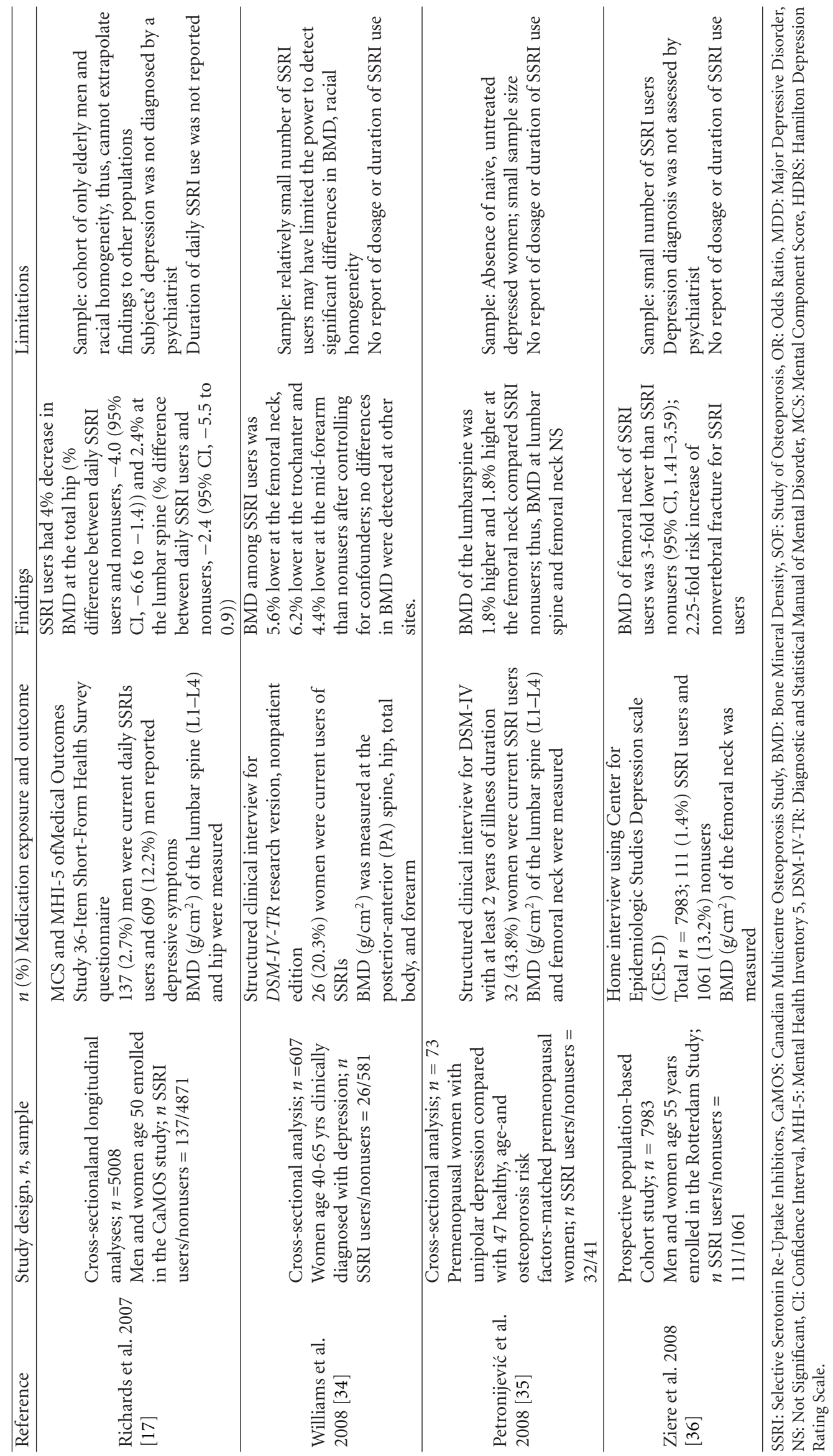


these 3 studies, a study conducted by Kavuncu et al. using a similar sample of patients taking SSRIs reported greater bone resorption [41].

\section{Limitations}

MDD affects as many as $16 \%$ of adult in the US [42], with prevalence increasing as the population ages [43]. Depression has also been linked to a reduced BMD in some $[16,17,32,34,36]$, but not all [31, 33, 35] studies. Therefore, a difficult task when defining the association between treatment with SSRI and lower BMD is controlling for confounding factors. Several clinical studies involving a population of depressed subjects to examine BMD in SSRI users and nonusers, however, have demonstrated that SSRIs may independently impact bone health, while physiologic and hormonal changes associated with depressive symptoms may magnify the adverse side effects of SSRI [16, 17, 32, $34,44,45]$. Therefore, it is possible that depression, in combination with SSRI treatment, may have an additive negative effect on BMD. Accordingly, an investigation of the potential contribution of mental illness a subsequent SSRI treatment as a determinant of bone health is warranted.

\section{Conclusion}

The vastly growing body of research on SSRIs and its effect on bone health suggests that this relationship is complex and interpreting these findings has proved to be challenging. Although multiple consistent findings reveal a trend suggesting that SSRI use may negatively impact bone and result in lower BMD, a definitive causal relationship cannot be drawn. The distinct fact that depression itself, both as a consequence of innate biological changes that accompany the illness and secondary to lifestyle factors such as poor diet and lack of activity that often are linked to depression, has been shown to cause bone loss poses depression as a confounding variable in epidemiologic studies investigating the exact effects of SSRIs on bone health. While it may be too soon to infer causality, however, the burgeoning mountain of evidence consistently demonstrating an association between SSRI use and bone loss now seems sufficient to consider adding SSRIs to the list of medications that contribute to osteoporosis. This would imply that clinicians consider bone density testing for people on SSRIs, or those on SSRIs with certain additional risk factors, for their risk of fracture. Further investigations are needed to confirm the serotoninergic effects on bone to definitely guide physicians to provide clear recommendations to patients regarding the clinical implications associated with SSRI treatment. It is also necessary to continue future investigations to definitely prove a casual connection between SSRI use and bone, and furthermore, to confirm the recent promising animal findings that may potentially prevent and treat bone loss.

\section{Conflict of Interests}

The authors declare that there is no conflict of interests.

\section{References}

[1] A. M. Sawka et al., "The number of Canadian men and women needed to be screened to detect a case of osteoporosis: a population based study from the Canadian Multicentre Osteoporosis Study (CaMos)," Journal of Bone Mineral Research, vol. 19, p. S368, 2004.

[2] A. C. Looker, L. J. Melton, T. B. Harris, and J. A. Shepherd, "Prevalence and trends in low femur bone density among older US adults: NHANES 2005-2006 compared with NHANES III," Journal of Bone and Mineral Research, vol. 25, no. 1, pp. 64-71, 2010.

[3] D. A. Doherty, K. M. Sanders, M. A. Kotowicz, and R. L. Prince, "Lifetime and five-year age-specific risks of first and subsequent osteoporotic fractures in postmenopausal women," Osteoporosis International, vol. 12, no. 1, pp. 16-23, 2001.

[4] S. R. Cummings, D. M. Blacks, and S. M. Rubin, "Lifetime risks of hip, Colles', or vertebral fracture and coronary heart disease among white postmenopausasl women," Archives of Internal Medicine, vol. 149, no. 11, pp. 2445-2448, 1989.

[5] L. J. Melton, E. A. Chrischilles, C. Cooper, A. W. Lane, and B. L. Riggs, "Perspective: how many women have osteoporosis?" Journal of Bone and Mineral Research, vol. 7, no. 9, pp. 10051010, 1992.

[6] G. Ioannidis, A. Papaioannou, W. M. Hopman et al., "Relation between fractures and mortality: results from the Canadian Multicentre Osteoporosis Study," Canadian Medical Association Journal, vol. 181, no. 5, pp. 265-271, 2009.

[7] M. E. Wiktorowicz, R. Goeree, A. Papaioannou, J. D. Adachi, and E. Papadimitropoulos, "Economic implications of hip fracture: health service use, institutional care and cost in Canada," Osteoporosis International, vol. 12, no. 4, pp. 271$278,2001$.

[8] WHO, "The world health report 2001: mental health: new understanding, new hope," Geneva, Switzerland, 2001.

[9] V. Taylor, M. C. McKinnon, K. Macdonald, G. Jaswal, and G. M. MacQueen, "Adults with mood disorders have an increased risk profile for cardiovascular disease within the first 2 years of treatment," Canadian Journal of Psychiatry, vol. 55, no. 6, pp. 362-368, 2010.

[10] J. J. Mann, K. M. Malone, D. J. Diehl, J. Perel, T. B. Cooper, and M. A. Mintun, "Demonstration in vivo of reduced serotonin responsivity in the brain of untreated depressed patients," American Journal of Psychiatry, vol. 153, no. 2, pp. 174-182, 1996.

[11] J. R. Raymond, Y. V. Mukhin, A. Gelasco et al., "Multiplicity of mechanisms of serotonin receptor signal transduction," Pharmacology and Therapeutics, vol. 92, no. 2-3, pp. 179-212, 2001.

[12] D. L. Murphy, A. Lerner, G. Rudnick, and K. P. Lesch, "Serotonin transporter: gene, genetic disorders, and pharmacogenetics," Molecular Interventions, vol. 4, no. 2, pp. 109-123, 2004.

[13] M. A. H. Rot, S. J. Mathew, and D. S. Charney, "Neurobiological mechanisms in major depressive disorder," Canadian Medical Association Journal, vol. 180, no. 3, pp. 305-313, 2009.

[14] R. Hubbard, P. Farrington, C. Smith, L. Smeeth, and A. Tattersfield, "Exposure to tricyclic and selective serotonin reuptake inhibitor antidepressants and the risk of hip fracture," American Journal of Epidemiology, vol. 158, no. 1, pp. 77-84, 2003.

[15] M. Kinjo, S. Setoguchi, S. Schneeweiss, and D. H. Solomon, "Bone mineral density in subjects using central nervous 
system-active medications," American Journal of Medicine, vol. 118, no. 12, p. 1414, 2005.

[16] S. J. Diem, T. L. Blackwell, K. L. Stone et al., "Use of antidepressants and rates of hip bone loss in older women: the study of osteoporotic fractures," Archives of Internal Medicine, vol. 167, no. 12, pp. 1240-1245, 2007.

[17] J. B. Richards, A. Papaioannou, J. D. Adachi et al., "Effect of selective serotonin reuptake inhibitors on the risk of fracture," Archives of Internal Medicine, vol. 167, no. 2, pp. 188-194, 2007.

[18] S. Z. Langer, N. Brunello, G. Racagni, and J. Mendlewicz, Serotonin Receptor Subtypes: Pharmacological Significance and Clinical Implications, Karger, Basel, Switzerland, 1992.

[19] B. E. Leonard, "Sub-types of serotonin receptors: biochemical changes and pharmacological consequences," International Clinical Psychopharmacology, vol. 7, no. 1, pp. 13-21, 1992.

[20] G. R. Heninger, "Indoleamines: the role of serotonin in clinical disorders," in Psycho- Pharmacology: The Fourth Generation of Progress, F. E. Bloom and D. J. Kupfer, Eds., pp. 471-482, Raven Press, New York, NY, USA, 1995.

[21] B. L. Jacobs and C. A. Fornal, "Serotonin and behavior: a general hypothesis," in Psychopharmacology: The Fourth Generation of Progress, F. E. Bloom and D. J. Kupfer, Eds., pp. 461-470, Raven Press, New York, NY, USA, 1995.

[22] S. M. Stahl, "Mechanism of action of serotonin selective reuptake inhibitors. Serotonin receptors and pathways mediate therapeutic effects and side effects," Journal of Affective Disorders, vol. 51, no. 3, pp. 215-235, 1998.

[23] M. M. Bliziotes, A. J. Eshleman, X. W. Zhang, and K. M. Wiren, "Neurotransmitter action in osteoblasts: expression of a functional system for serotonin receptor activation and reuptake," Bone, vol. 29, no. 5, pp. 477-486, 2001.

[24] I. Westbroek, A. Van der Plas, K. E. De Rooij, J. Klein-Nulend, and P. J. Nijweide, "Expression of serotonin receptors in bone," The Journal of Biological Chemistry, vol. 276, no. 31, pp. 28961-28968, 2001.

[25] R. Battaglino, J. Fu, U. Späte et al., "Serotonin regulates osteoclast differentiation through its transporter," Journal of Bone and Mineral Research, vol. 19, no. 9, pp. 1420-1431, 2004.

[26] S. J. Warden, M. M. Bliziotes, K. M. Wiren, A. J. Eshleman, and C. H. Turner, "Neural regulation of bone and the skeletal effects of serotonin (5-hydroxytryptamine)," Molecular and Cellular Endocrinology, vol. 242, no. 1-2, pp. 1-9, 2005.

[27] P. Vestergaard, L. Rejnmark, and L. Mosekilde, "Anxiolytics, sedatives, antidepressants, neuroleptics and the risk of fracture," Osteoporosis International, vol. 17, no. 6, pp. 807-816, 2006.

[28] L. Spangler, D. Scholes, R. L. Brunner et al., "Depressive symptoms, bone loss, and fractures in postmenopausal women," Journal of General Internal Medicine, vol. 23, no. 5, pp. 567574, 2008.

[29] R. Yirmiya and I. Bab, "Major depression is a risk factor for low bone mineral density: a meta-analysis," Biological Psychiatry, vol. 66, no. 5, pp. 423-432, 2009.

[30] V. K. Yadav, F. Oury, N. Suda et al., "A Serotonin-dependent mechanism explains the leptin regulation of bone mass, appetite, and energy expenditure," Cell, vol. 138, no. 5, pp. 976-989, 2009.

[31] D. Michelson, C. Stratakis, L. Hill et al., "Bone mineral density in women with depression," The New England Journal of Medicine, vol. 335, no. 16, pp. 1176-1181, 1996.

[32] J. A. Cauley, R. L. Fullman, K. L. Stone et al., "Factors associated with the lumbar spine and proximal femur bone mineral density in older men," Osteoporosis International, vol. 16, no. 12, pp. 1525-1537, 2005.

[33] F. Eskandari, P. E. Martinez, S. Torvik et al., "Low bone mass in premenopausal women with depression," Archives of Internal Medicine, vol. 167, no. 21, pp. 2329-2336, 2007.

[34] L. J. Williams, M. J. Henry, M. Berk et al., "Selective serotonin reuptake inhibitor use and bone mineral density in women with a history of depression," International Clinical Psychopharmacology, vol. 23, no. 2, pp. 84-87, 2008.

[35] M. Petronijević, N. Petronijević, M. Ivković et al., "Low bone mineral density and high bone metabolism turnover in premenopausal women with unipolar depression," Bone, vol. 42, no. 3, pp. 582-590, 2008.

[36] G. Ziere, J. P. Dieleman, T. J. M. Van Der Cammen, A. Hofman, H. A. P. Pols, and B. H. C. Stricker, "Selective serotonin reuptake inhibiting antidepressants are associated with an increased risk of nonvertebral fractures," Journal of Clinical Psychopharmacology, vol. 28, no. 4, pp. 411-417, 2008.

[37] C. J. L. Murray and A. D. Lopez, "Global mortality, disability, and the contribution of risk factors: global burden of disease study," The Lancet, vol. 349, no. 9063, pp. 1436-1442, 1997.

[38] J. W. Williams Jr., C. D. Mulrow, E. Chiquette, P. H. Noël, C. Aguilar, and J. Cornell, "A systematic review of newer pharmacotherapies for depression in adults: evidence report summary," Annals of Internal Medicine, vol. 132, no. 9, pp. 743-756, 2000.

[39] P. A. Pirraglia, R. S. Stafford, and D. E. Singer, "Trends in prescribing of selective serotonin reuptake inhibitors and other newer antidepressant agents in adult primary care," The Journal of Clinical Psychiatry, the Primary Care Companion, vol. 5, no. 4, pp. 153-157, 2003.

[40] I. A. Bab and R. Yirmiya, "Depression and bone mass," Annals of the New York Academy of Sciences, vol. 1192, pp. 170-175, 2010.

[41] V. Kavuncu, M. Kuloglu, A. Kaya, S. Sahin, M. Atmaca, and B. Firidin, "Bone metabolism and bone mineral density in premenopausal women with mild depression," Yonsei Medical Journal, vol. 43, no. 1, pp. 101-108, 2002.

[42] R. C. Kessler, M. Angermeyer, J. C. Anthony et al., "Lifetime prevalence and age-of-onset distributions of mental disorders in the world health organization's world mental health survey initiative," World Psychiatry, vol. 6, no. 3, pp. 168-176, 2007.

[43] M. A. Whooley, K. E. Kip, J. A. Cauley, K. E. Ensrud, M. C. Nevitt, and W. S. Browner, "Depression, falls, and risk of fracture in older women," Archives of Internal Medicine, vol. 159, no. 5, pp. 484-490, 1999.

[44] B. Liu, G. Anderson, N. Mittmann, T. To, T. Axcell, and N. Shear, "Use of selective serotonin-reuptake inhibitors or tricyclic antidepressants and risk of hip fractures in elderly people," The Lancet, vol. 351, no. 9112, pp. 1303-1307, 1998.

[45] E. M. Haney, B. K. S. Chan, S. J. Diem et al., "Association of low bone mineral density with selective serotonin reuptake inhibitor use by older men," Archives of Internal Medicine, vol. 167, no. 12, pp. 1246-1251, 2007. 


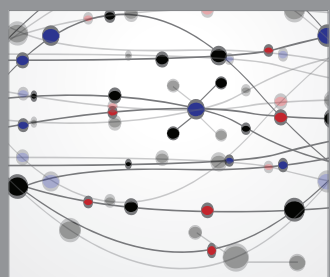

The Scientific World Journal
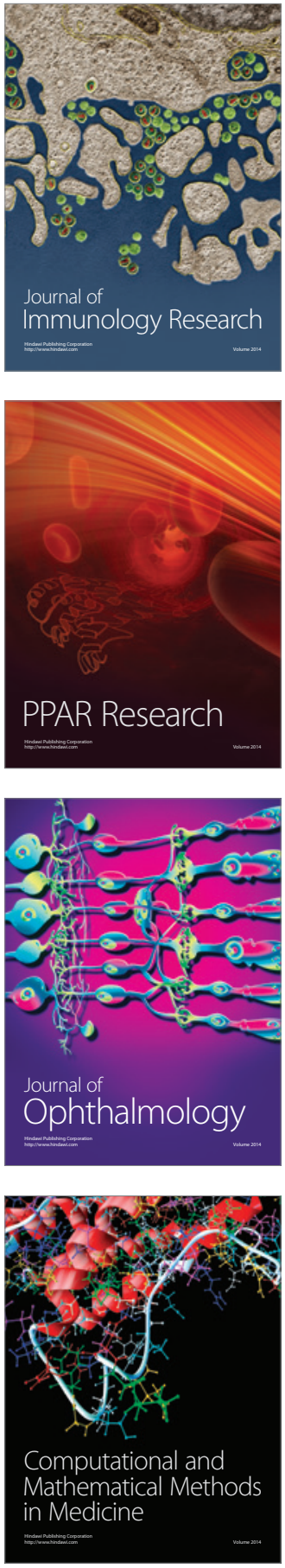

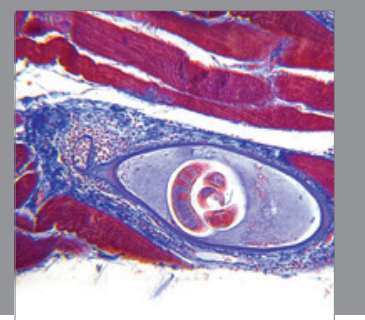

Gastroenterology

Research and Practice
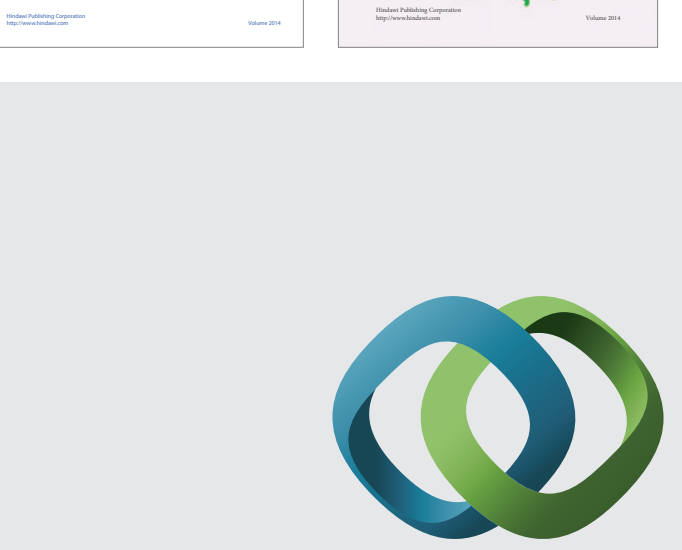

\section{Hindawi}

Submit your manuscripts at

http://www.hindawi.com
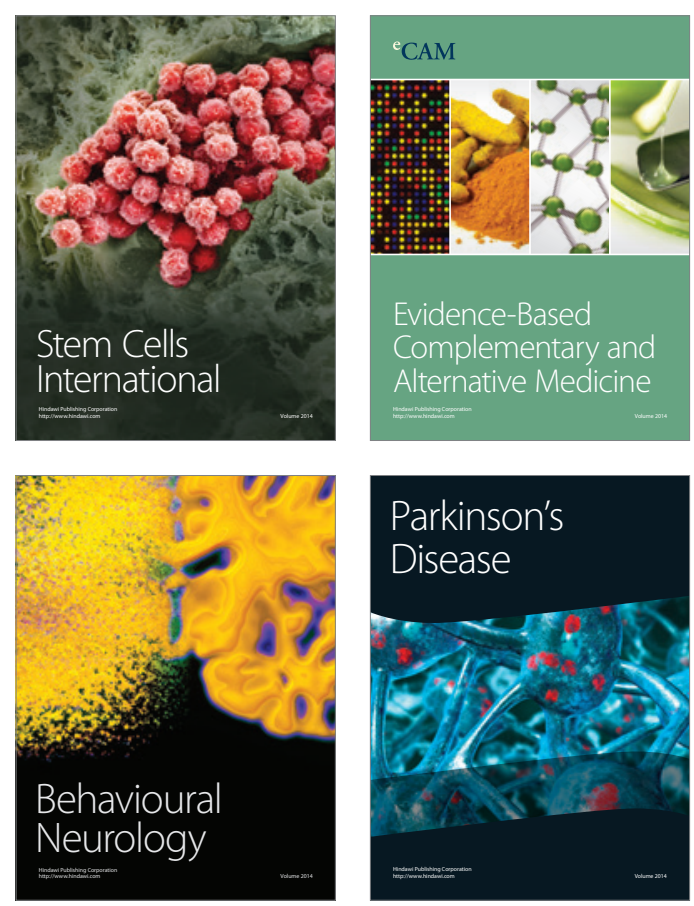

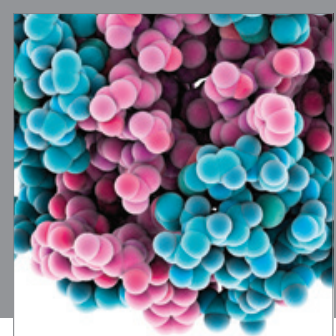

Journal of
Diabetes Research

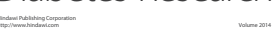

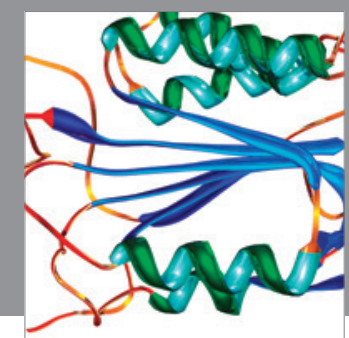

Disease Markers
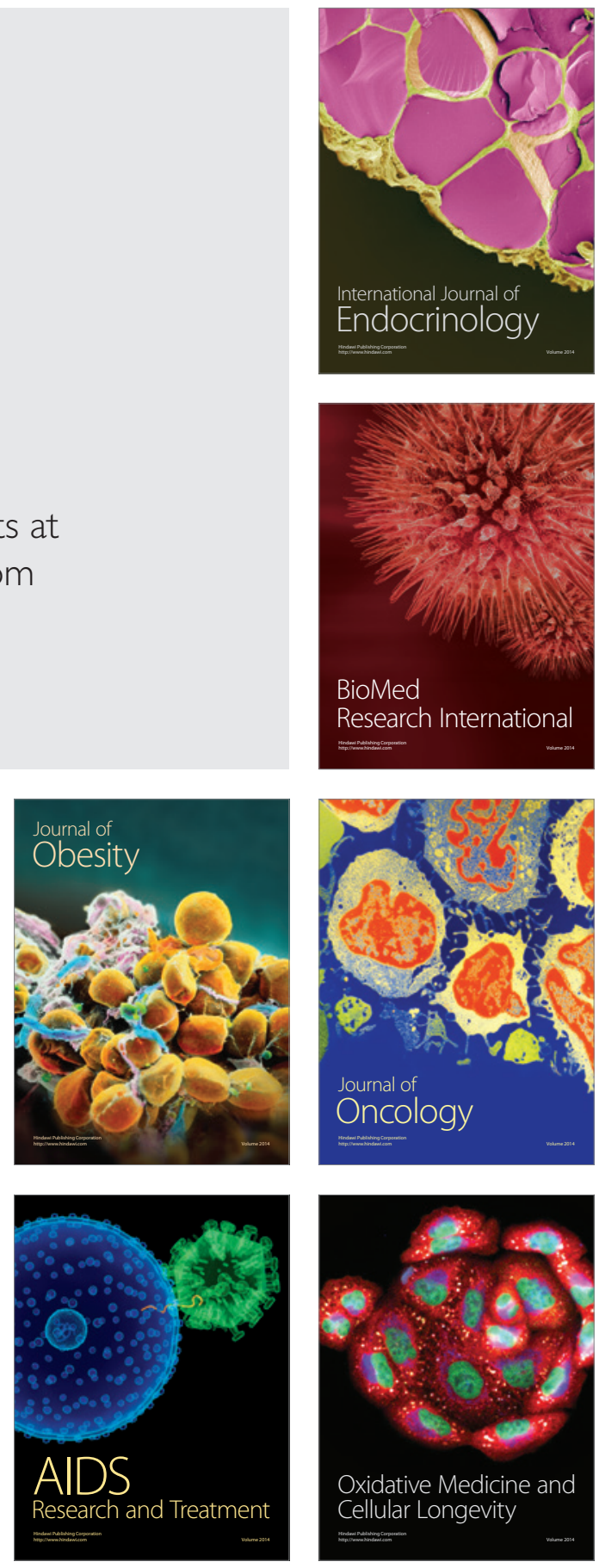